\title{
On cluster primes
}

\author{
by \\ Christian Elsholtz (Clausthal-Zellerfeld)
}

1. Introduction. Blecksmith, Erdős and Selfridge [1] defined a prime $p>2$ to be a cluster prime if every positive even integer $2 r \leq p-3$ can be written as a difference of two primes, $2 r=q-q^{\prime}$, where $q^{\prime} \leq q \leq p$. It is an open question whether there exist infinitely many cluster primes. Guy ([4, Section C1]) attributes this question to Erdös. The attention of the general audience was drawn to this problem by Peterson's article [6] in Science News.

Blecksmith et al. [1] proved that the counting function $\pi_{C}(x)$ of cluster primes can be bounded from above: for all positive $s$,

$$
\pi_{C}(x)=O_{s}\left(\frac{x}{(\log x)^{s}}\right) .
$$

It is the purpose of this note to prove a better bound, i.e. that cluster primes are rare. This new bound was indeed conjectured by Blecksmith et al. [1].

ThEOREM. The number $\pi_{C}(x)$ of cluster primes below $x$ is bounded by

$$
\pi_{C}(x)=O\left(\frac{x}{\exp \left(\frac{1}{60}(\log \log x)^{2}\right)}\right) .
$$

As Blecksmith, Erdôs and Selfridge show, the problem is related to the prime $k$-tuple conjecture. It is proved that for a cluster prime $p$ the interval $[p-t, p)$ must contain sufficiently many primes, which explains the name cluster prime. This allows us to apply an upper bound sieve. In Blecksmith et al. [1], Brun's version of the small sieve is used. The principal problem is that the authors arrive at a constant $M$ whose dependence on the sieve dimension $s$ is not at all clear. This prohibits taking an increasing $s$.

Filaseta [3] mentioned that an application of Hooley's almost pure sieve proves the result with $s=\varepsilon \log \log \log x$, thus obtaining an upper bound of $\pi_{C}(x)=O\left(\frac{x}{\exp (a \log \log x \log \log \log x)}\right) \quad$ for some positive constant $a$.

2000 Mathematics Subject Classification: Primary 11N05, 11N36; Secondary 11A41. Key words and phrases: prime tuples, sieve methods. 
In this note we apply the large sieve method, due to Montgomery [5]. In fact, we make use of the following lemma due to Vaughan [7], which is an elaborated version of the large sieve method, perfectly fitting to our application.

Lemma 1 (Montgomery [5], Vaughan [7]). Denote by $\mathcal{P}$ the set of primes and let $\omega: \mathcal{P} \rightarrow \mathbb{N}$ with $0 \leq \omega(p) \leq p-1$. Let $\mathcal{A} \subset[1, N]$ denote a set of integers which lies outside $\omega(p)$ residue classes modulo the prime $p$. Then the number $A(x)$ of elements $n \in \mathcal{A}$ with $n \leq x$ satisfies

$$
A(x) \leq \frac{2 x}{L}, \quad \text { where } L=\sum_{q \leq x^{1 / 2}} \mu^{2}(q) \prod_{p \mid q} \frac{\omega(p)}{p-\omega(p)} .
$$

Moreover,

$$
L \geq \max _{m \in \mathbb{N}} \exp \left(m \log \left(\frac{1}{m} \sum_{p \leq x^{1 /(2 m)}} \frac{\omega(p)}{p}\right)\right) .
$$

2. Proof of the Theorem. If $p$ is a cluster prime, then the even integers like $p-9$ or $p-15$ are the differences of two primes $q, q^{\prime}$ with $q, q^{\prime} \leq p$. In particular, there must be a prime in the interval $[p-6, p)$. More generally, an even integer $2 r \in[p-t, p-3]$ must be represented by a prime $q \in[p-t, p]$ and a prime $q^{\prime} \in[1, t]$. By the prime number theorem the number of primes in $[1, t]$ is $(1+o(1)) t / \log t$. We see that for any $\varepsilon>0$ there must be at least $s:=(1 / 2-\varepsilon) \log t$ primes in $[p-t, p)$.

Since the average gap between primes of size $x$ is about $\log x$ we see that this is a useful criterion for $t=O\left((\log x)^{\delta}\right)$ (with $\left.0<\delta<1\right)$. On the contrary, for sufficiently large $t$ one expects that an interval of length $t$ has about $t / \log t$ primes so that this criterion becomes useless.

There are (trivially) at most $\left(\begin{array}{l}t \\ s\end{array}\right)$ possibilities to place $s$ primes in an interval of length $t$. For any pattern of $s$ primes in $[p-t, p)$ we will give an upper bound on the number of prime $s$-tuples below $x$. This bound will not depend on the particular pattern. So, multiplying this bound by the upper bound for the number of patterns, $\left(\begin{array}{l}t \\ s\end{array}\right)$, gives an upper bound on the number of $p \leq x$ such that the interval $[p-t, p)$ contains (at least) $s$ primes.

We prove the following lemma:

LEMMA 2. Let $\delta=1 /(2 e)$ and $t=(\log x)^{\delta}$. Let $\varepsilon$ be a sufficiently small positive constant. Let $A(x)$ denote the number of integers $n \leq x$ such that the interval $[n-t, n)$ contains at least $s=(1 / 2-\varepsilon) \log t$ primes. Then

$$
A(x)=O\left(\frac{x}{\exp \left(\left(1 /\left(4 e^{2}\right)-\varepsilon\right)(\log \log x)^{2}\right)}\right) .
$$


We fix a particular pattern $a_{1}<\ldots<a_{s}$. If all $n-a_{i}$ are prime simultaneously, then the integers $n$ avoid the residue classes $a_{i} \bmod p$ for $p \leq n-t$. If $p>t$, then the number of forbidden classes is $\omega(p)=s$.

We choose $m=\left\lceil\delta^{2}(\log \log x)^{2}\right\rceil$, where $x$ is large. With $y=x^{1 /(2 m)}$ we have $\log \log y=\log \log x-2 \log \log \log x+O(1)$. So we find that

$$
\begin{aligned}
\sum_{p \leq y} \frac{\omega(p)}{p} & \geq \sum_{t \leq p \leq y} \frac{\omega(p)}{p} \geq s(\log \log y-\log \log t+o(1)) \\
& \geq(1 / 2-\varepsilon) \delta(\log \log x)(\log \log x-3 \log \log \log x+O(1)) \\
& \geq(1 / 2-2 \varepsilon) \delta(\log \log x)^{2} .
\end{aligned}
$$

This implies the estimate

$$
\begin{aligned}
L & \geq \exp \left(\left\lceil\delta^{2}(\log \log x)^{2}\right\rceil \log \left(\frac{1}{\left\lceil\delta^{2}(\log \log x)^{2}\right\rceil} \delta\left(\frac{1}{2}-2 \varepsilon\right)(\log \log x)^{2}\right)\right) \\
& \geq \exp \left(\delta^{2}(\log \log x)^{2} \log \left(\frac{1}{\delta}\left(\frac{1}{2}-3 \varepsilon\right)\right)\right) \\
& \geq \exp \left(\delta^{2}(\log \log x)^{2} \log (e-6 e \varepsilon)\right) \geq \exp \left(\delta^{2}(1-7 \varepsilon)(\log \log x)^{2}\right) \\
& \geq \exp \left(\left(\frac{1}{4 e^{2}}-\varepsilon\right)(\log \log x)^{2}\right) .
\end{aligned}
$$

Therefore, for any fixed pattern $a_{1}<\ldots<a_{s}$ there are at most

$$
\frac{2 x}{\exp \left(\left(1 /\left(4 e^{2}\right)-\varepsilon\right)(\log \log x)^{2}\right)}
$$

values $n \leq x$ such that all $n-a_{i}$ are prime. Thus the lemma is proved.

To prove the theorem we only need to recall that

Because of

$$
\pi_{C}(x) \leq\left(\begin{array}{l}
t \\
s
\end{array}\right) \frac{2 x}{\exp \left(\delta^{2}(1-7 \varepsilon)(\log \log x)^{2}\right)} .
$$

$$
\left(\begin{array}{l}
t \\
s
\end{array}\right) \leq t^{s} \leq \exp \left((1 / 2-\varepsilon) \delta^{2}(\log \log x)^{2}\right)
$$

we find that

$$
\pi_{C}(x)=O\left(\frac{x}{\exp \left(\left(1 /\left(8 e^{2}\right)-\varepsilon\right)(\log \log x)^{2}\right)}\right) .
$$

3. Further comments. No serious attempt has been made at optimizing the constant $1 / 60$ or $1 /\left(8 e^{2}\right)-\varepsilon$ that appears in the Theorem. Some improvement is possible. We only mention the following: Vaughan's argument in Lemma 1 can be refined to

$$
L \geq \max _{m \in \mathbb{N}} \exp \left(m \log \left(\frac{e-\varepsilon_{m}}{m} \sum_{p \leq x^{1 /(2 m)}} \frac{\omega(p)}{p}\right)\right) .
$$


Here the $\varepsilon_{m}$ are positive constants that tend to 0 as $m$ goes to infinity. This allows using $c_{1} \approx \delta / 2$ and $\delta \approx 1 / 2$ and proves the Theorem with $1 / 8-\varepsilon$ instead of $1 / 60$. For details see [2].

The author would like to thank the referees for helpful comments.

\section{References}

[1] R. Blecksmith, P. Erdős and J. L. Selfridge, Cluster primes, Amer. Math. Monthly 106 (1999), 43-48.

[2] C. Elsholtz, Combinatorial prime number theory - a study of the gap structure of the set of primes, Habilitationsschrift, Technische Universität Clausthal, 2002.

[3] M. Filaseta, Review of [1] in the Mathematical Reviews: 2000a:11126.

[4] R. K. Guy, Unsolved Problems in Number Theory, 2nd ed., Springer, New York, 1994.

[5] H. L. Montgomery, The analytic principle of the large sieve, Bull. Amer. Math. Soc. 84 (1978), 547-567.

[6] I. Peterson, The scarcity of cluster primes, Science News 6, February 1999, p. 95.

[7] R. C. Vaughan, Some applications of Montgomery's sieve, J. Number Theory 5 (1973), 64-79.

Institut für Mathematik

TU Clausthal

Erzstrasse 1

D-38678 Clausthal-Zellerfeld, Germany

E-mail: elsholtz@math.tu-clausthal.de

Received on 27.5.2002

and in revised form on 26.2.2003 